\title{
Quantized conductance with nonzero shot noise as a signature of Andreev edge state
}

\author{
Manas Ranjan Sahu, ${ }^{1, *}$ Arup Kumar Paul, ${ }^{1,}$ Jagannath Sutradhar, ${ }^{1, *}$ K. Watanabe $\odot,{ }^{2}$ T. Taniguchi, ${ }^{2}$ Vibhor Singh, \\ Subroto Mukerjee, ${ }^{1}$ Sumilan Banerjee, ${ }^{1, \dagger}$ and Anindya Das $\oplus^{1, \ddagger}$ \\ ${ }^{1}$ Department of Physics, Indian Institute of Science, Bangalore 560012, India \\ ${ }^{2}$ National Institute for Materials Science, 1-1 Namiki, Tsukuba 305-0044, Japan
}

(Received 10 March 2021; accepted 11 August 2021; published 18 August 2021)

\begin{abstract}
Electrical conductance measurements have a limited scope in identifying Andreev edge states (AESs), which form the basis for realizing various topological excitations in quantum Hall (QH)-superconductor (SC) junctions. To unambiguously detect AESs, we measure shot noise along with electrical conductance in a graphene-based QH-SC junction at integer filling $v=2$. Remarkably, we find that the Fano factor of shot noise approaches half when the bias energy is less than the superconducting gap $(2 \Delta)$, whereas it is close to zero above $2 \Delta$. This is striking, given that, at the same time, the electrical conductance remains quantized at $2 e^{2} / h$ within and above $2 \Delta$. A quantized conductance is expected to produce zero-shot noise due to its dissipationless flow. However, at a QH-SC interface, AESs carry the current in the zero-bias limit and an equal mixing of electron- and holelike states produces half of the Poissonian shot noise with quantized conductance. The observed results are in accord with our detailed theoretical calculations of electrical conductance and shot noise based on the nonequilibrium Green's function method in the presence of disorder. Our results pave the way in using shot noise as a detection tool in the search for exotic topological excitations in QH-SC hybrids.
\end{abstract}

DOI: 10.1103/PhysRevB.104.L081404

Chiral quantum Hall $(\mathrm{QH})$ edge states in proximity to a superconductor (SC) can give rise to exotic excitations [1-6] such as Majorana fermions. There are several promising theoretical proposals of realizing chiral Majorana fermions at the QH-SC interfaces [4,5,7], however, evidence is still inconclusive [8-11]. The realization of electron-hole hybrid states called Andreev edge states (AESs) [12,13] at the QH-SC interface is an important step in this quest and graphene hosting clean $\mathrm{QH}$ edge states at a moderate magnetic field is an ideal platform. The recent developments of several superconductors with a large critical magnetic field and transparent interfaces with high quality graphene have paved the way for a number of interesting experimental observations [14-26], such as crossed Andreev conversion [18], supercurrents in the QH regime [16], inter-Landau-level Andreev reflection [19], and interference of chiral AESs [21]. Despite this progress, the identification of AESs remains scarce, and its dynamics have remained unexplored in the presence of disorder and dissipation.

AESs result from the repeated Andreev reflections at a $\mathrm{QH}-$ SC interface, where an incident electron successively turns into a hole and back into an electron. Quantum mechanically, AESs are fermionic modes that arise from linear combinations of electrons and holes, and propagate along the interface as shown schematically in Fig. 1(a). The nature of the resultant fermion coming out from the interface relies on the interference of the AESs. An electron or hole or a mixture of electrons

\footnotetext{
*These authors contributed equally to this work.

†sumilan@iisc.ac.in

‡anindya@iisc.ac.in
}

and holes can exit at the end of the interface depending on the phase difference accumulated by the AESs. Thus, in a conventional conductance measurement, the conductance is expected to oscillate between zero and $4 e^{2} / h$ at a filling factor $v=2$ as a function of parameters such as the chemical potential and magnetic field, giving a robust signature of AESs. However, the inevitable presence of disorder and interface roughness randomizes the phase. As a result, a low visibility of conductance oscillations around $2 e^{2} / h$ is observed experimentally for shorter QH-SC interfaces [21]. For a wider QH-SC interface, the oscillations are expected to vanish due to complete phase averaging and will exhibit quantized conductance of $2 e^{2} / h$, exactly as a $\mathrm{QH}$-normal metal (NM) junction. Hence, the conductance measurement is a limited tool to explore the AESs at QH-SC interfaces. On the contrary, as we demonstrate here, the shot noise, which originates from the discrete nature of the current carriers [27-29], does not vanish due to the phase averaging and can provide new insights into the AES interference. The shot noise of a system is quantified by the Fano factor $(F)$, namely the ratio of measured current noise with the Poissonian value of the shot noise, 2eI, for a current $I$ [22,30-34]. Remarkably, we experimentally observe a large nonzero Fano factor, close to half, simultaneously with a quantized conductance in a graphene $\mathrm{QH}-\mathrm{SC}$ junction, thus providing a robust and unambiguous signature of AESs.

To this end, we perform conductance and shot noise measurements in a graphene QH-SC junction at filling factor $v=2$ as a function of excitation or bias energy $\left(e V_{S D}\right)$ at several magnetic fields $(B)$. As shown in Fig. 1(b), our shot noise results can be divided into three regions: bulk dominated transport at lower $B$, AES dominated transport at intermediate $B$, and normal quasiparticle dominated transport at higher $B$. 

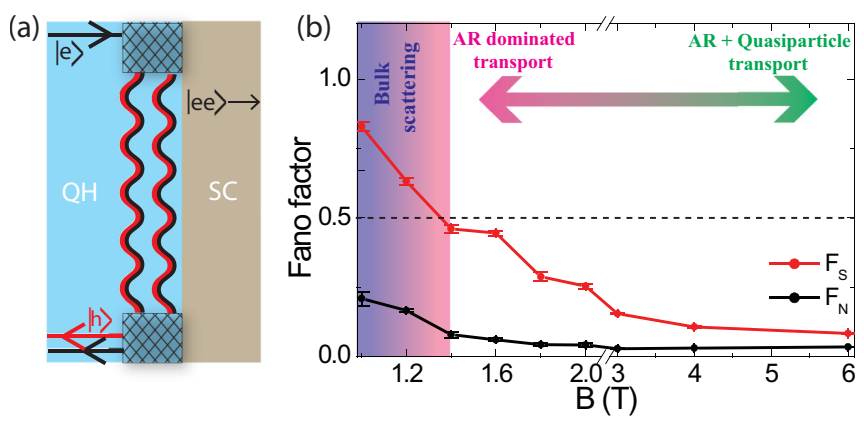

FIG. 1. (a) Schematic of the AESs (wavy lines). (b) Experimentally measured low-bias Fano factors $F_{S}$ and high-bias Fano factors $F_{N}$ are plotted as a function of magnetic field. The different transport regimes are highlighted by the gradient colors. The dashed horizontal line corresponds to half Fano.

In the intermediate $B$, the Fano factor approaches a value close to half when $e V_{S D} \ll 2 \Delta$, whereas the Fano factor is close to zero for normal quasiparticle transport for $e V_{S D} \gg$ $2 \Delta$ [Fig. 1(b)]. In contrast, the conductance always remains $2 e^{2} / h$ across the excitation energy $2 \Delta$. Our experimental observations are supported by an effective model [Fig. 1(a)], discussed later, which predicts a Fano factor of half for a $\mathrm{QH}$ $\mathrm{SC}$ junction for a complete phase averaging of the interference of AESs. This is in stark contrast to the zero Fano factor expected for a QH-NM junction. Moreover, we also calculate the conductance and Fano factor for a microscopic model of a graphene QH-SC junction in the presence of disorder using the nonequilibrium Green's function (NEGF) method. We find disorder averaged conductance $(G)$ with quantized plateaus $\left(v e^{2} / h\right)$, as in a QH-NM junction, and a large nonzero quantized Fano factor for a $v=2$ plateau as a consequence of equal mixing of electron- and holelike states.

To realize the coupling of $\mathrm{QH}$ and superconductivity, we fabricated a hexagonal boron nitride (hBN) encapsulated graphene device edge contacted with a type-II molybdenum rhenium (MoRe) superconductor on a $\mathrm{Si} / \mathrm{SiO}_{2}$ substrate. The width of the graphene-SC interface was $\sim 2 \mu \mathrm{m}$ and the channel length was $\sim 0.8 \mu \mathrm{m}$. Figure 2(a) shows the schematic of the device with the measurement setup. The device fabrication and measurement setup are discussed in detail in the Supplemental Material (SM) [35]. The MoRe leads show a superconducting transition at $T_{c} \sim 8.7 \mathrm{~K}$ (Fig. S5). Figure 2(b) shows the supercurrent of the MoRe-grapheneMoRe junction at zero magnetic field demonstrating the high quality of graphene-MoRe interfaces (see details in SM Sec. S8 [35]). The supercurrent of the junction is killed by applying a tiny field of $100 \mathrm{mT}$, whereas the MoRe leads remain superconducting at large magnetic fields as shown by the $I-V$ characteristic in Fig. 2(c) depicting a supercurrent at $B=8 \mathrm{~T}$.

Clean QH plateaus of the MoRe-graphene-MoRe junction are observed at magnetic fields as low as $1 \mathrm{~T}$ (Fig. S6). Figure 2(d) shows two-probe conductance $(G)$ around $v=2$ filling at $B=2 \mathrm{~T}$ as a function of back-gate voltage $\left(V_{\mathrm{BG}}\right)$ and the conductance plateau remains very close to $2 e^{2} / h$, similar to the QH-NM interface. Further, the conductance remains almost unchanged by the application of bias energy as shown in the inset of Fig. 2(d). The vertical dashed lines mark the
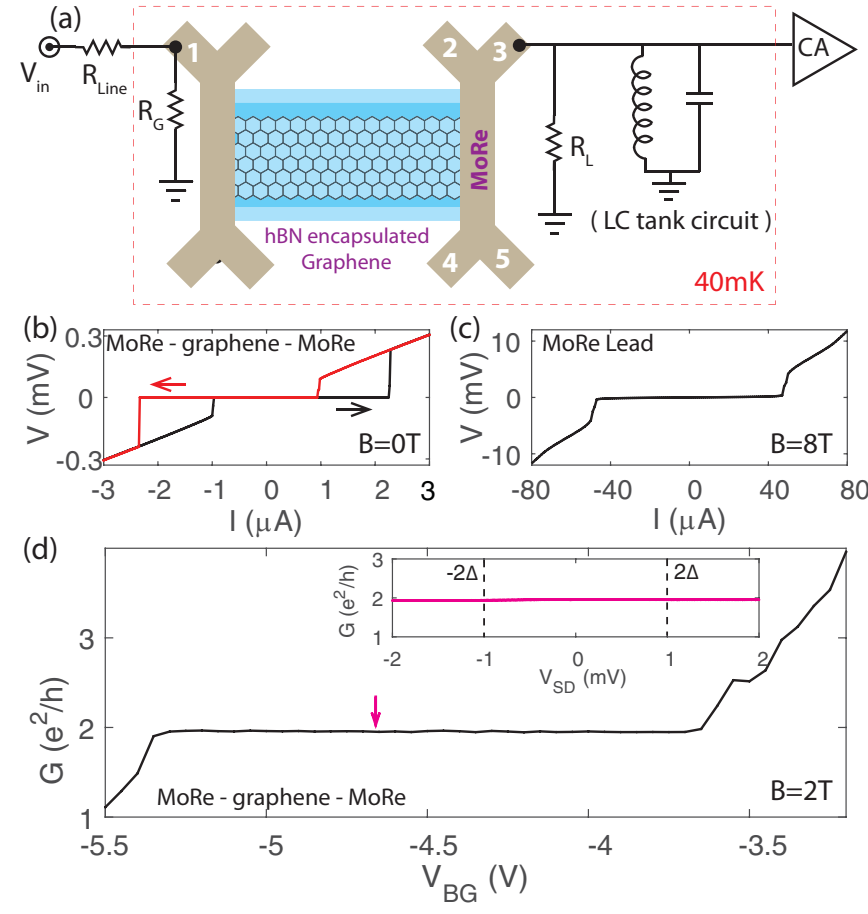

FIG. 2. (a) The schematic of the MoRe-graphene-MoRe device along with the shot noise measurement setup. (b) The $I-V$ response of the MoRe-graphene-MoRe device showing supercurrent at zero magnetic field. (c) The $I-V$ response of the MoRe lead showing supercurrent at $B=8 \mathrm{~T}$. (d) The conductance of the MoRe-graphene-MoRe device at the $v=2 \mathrm{QH}$ plateau at $B=2 \mathrm{~T}$ plotted as a function of gate voltage. The inset shows conductance at $v=2$ with bias energy. The vertical lines correspond to the superconducting gap.

proximity induced superconducting gap, $2 \Delta \sim 1 \mathrm{meV}$, as can be seen from our shot noise data discussed later. The conductance values for the $v=2$ and $v=6$ filling factors remain very close to $2 e^{2} / h$ and $6 e^{2} / h$, respectively, for the full range of magnetic fields from 1 to $10 \mathrm{~T}$ (Fig. S6). These observations are in accordance with recent experiments [18,21]. However, there were no noticeable oscillations at $v=2$ [Fig. 2(d)] as compared to the oscillations observed by Zhao et al. [21].

Now we present the shot noise data $\left(S_{I}\right)$ of the device. Figure 3(a) shows the shot noise data taken at the center of the $v=2 \mathrm{QH}$ plateau plotted as a function of applied DC current $\left(I_{S D}\right)$ at several magnetic fields. Interestingly, $S_{I}$ does not increase linearly for the full range of current. Rather, a local linearity can be seen either at a lower current $(<20 \mathrm{nA})$ or at larger current $(>100 \mathrm{nA})$ with the slope being significantly larger for the former case. To better understand this, we plot the differential Fano factor, $F=(1 / 2 e)\left(d S_{I} / d I_{S D}\right)$, for $B=1.6 \mathrm{~T}$ as a function of $I_{S D}$ in Fig. 3(b). The differential Fano factor peaks around zero $I_{S D}$ and saturates to a very small magnitude at larger $I_{S D}$. Such a transition is expected around the superconducting gap $(2 \Delta \sim 1 \mathrm{meV})$ marked by the vertical dashed lines. The observed gap is smaller than the expected BCS gap $2 \Delta_{\mathrm{BCS}} \sim 2 \mathrm{meV}$ as calculated from the critical temperature $(\sim 7-8 \mathrm{~K})$ of the bulk MoRe leads. Such a reduction was observed in the past and was attributed to the superconducting proximity effect $[36,37]$. We define a 

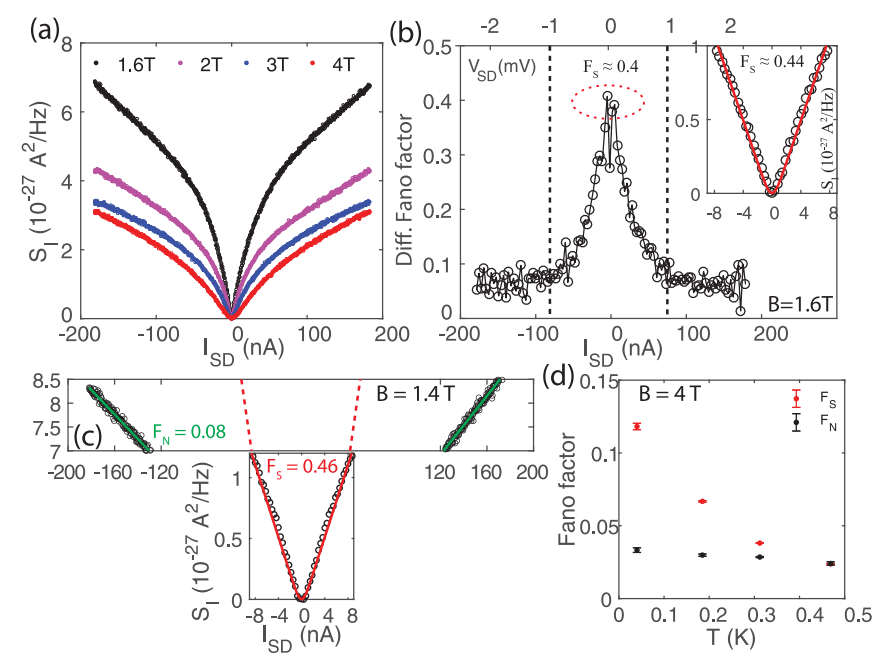

FIG. 3. (a) The measured $S_{I}$ plotted as a function of $I_{S D}$ at the center of the $v=2$ plateau at $40 \mathrm{mK}$ for several $B$. (b) Differential Fano for the $v=2(B=1.6 \mathrm{~T})$ plateau plotted as a function of $I_{S D}$. The top axis shows the corresponding excitation voltage $\left(V_{S D}\right)$. The two vertical dashed lines mark the approximate proximity induced superconducting gap $2 \Delta \sim 1 \mathrm{meV}$. The inset shows the low-bias fitting using Eq. (1) giving $F_{S} \sim 0.44$. (c) Low- $\left(e V_{S D} \ll 2 \Delta\right)$ and high-bias $\left(e V_{S D} \gg 2 \Delta\right)$ fittings are shown for the shot noise data at the $v=2 \mathrm{QH}$ plateau at $B=1.4 \mathrm{~T}$. (d) $F_{S}$ and $F_{N}$ for the $v=2 \mathrm{QH}$ plateau at $B=4 \mathrm{~T}$ as a function of temperature.

low-bias Fano factor $F_{S}$ for $e V_{S D}<2 \Delta$, anticipating transport via Andreev reflections, whereas the high-bias Fano factor for $e V_{S D}>2 \Delta$ is denoted as $F_{N}$, which is expected to be very small since the transport occurs via normal quasiparticles. From the differential Fano factor we indeed find $F_{S}$ to be $\sim 0.4$ near zero bias and $F_{N}$ to saturate around $\sim 0.05$.

A more accurate estimation of $F_{S}$ and $F_{N}$ requires the fitting of $S_{I}$ data with the finite-temperature expression of shot noise $[22,27,38]$,

$$
S_{I}= \begin{cases}2 e I_{S D} F_{S}\left[\operatorname{coth}\left(\frac{e^{*} V_{S D}}{2 k_{B} T_{e}}\right)-\frac{2 k_{B} T_{e}}{e^{*} V_{S D}}\right], & \text { for }\left|e V_{S D}\right|<2 \Delta, \\ K+2 e I_{S D} F_{N}, & \text { for }\left|e V_{S D}\right|>2 \Delta,\end{cases}
$$

where $V_{S D}=\frac{I_{S D}}{G}$ is the excitation voltage, $k_{B}$ is the Boltzmann constant, and $T_{e}(\sim 40 \mathrm{mK})$ is the electron temperature. The inset of Fig. 3(b) shows the low-bias fitting of the $B=1.6 \mathrm{~T}$ shot noise data, which gives $F_{S}$ to be $\sim 0.44$. We extracted Fano factors for different magnetic fields by fitting the corresponding shot noise data with Eq. (1) as shown in Fig. 3(c) for $B=1.4 \mathrm{~T}$. The extracted $F_{S}$ and $F_{N}$ are plotted as a function of $B$ in Fig. 1(b), where it can be seen that $F_{N}$ remains almost constant $(\sim 0.05)$ at magnetic fields as low as $1.4 \mathrm{~T}$ to high magnetic fields. However, it increases rapidly below $1.4 \mathrm{~T}$ due to the bulk contribution. The presence of a bulk contribution below $1.4 \mathrm{~T}$ is further justified from the rapid degradation of the quality of the $v=2 \mathrm{QH}$ plateau in this regime, which is shown in Fig. S14. More interestingly, $F_{S}$ has a larger value and approaches close to half in the range 1.4-1.6 $\mathrm{T}$ and then decreases with increasing magnetic field. In SM Sec. S5 [35], we discuss how the presence of vortices at the QH-SC interface can enable normal quasiparticle transport and hence reduce the value of the Fano factor with increasing magnetic field. In Fig. 3(d), we show $F_{S}$ and $F_{N}$ for the $v=2 \mathrm{QH}$ plateau with the temperature at $B=4 \mathrm{~T}$. It can be seen that $F_{S}$ decreases with increasing temperature and becomes almost equal to $F_{N}$ at $\sim 0.5 \mathrm{~K}$ due to normal quasiparticle transport. The $T \sim 0.5 \mathrm{~K}$ is much smaller than the $T_{C} \sim 7-8 \mathrm{~K}$ of the bulk MoRe lead. This reduction in temperature is possibly due to the superconducting proximity effect and is discussed in detail in SM Sec. S9 [35]. Note that, for normal transport via QH edge states, no shot noise is expected as the transmission probability is equal to one. For a junction with reduced transparency $\left(T_{N}<1\right)$ the shot noise can be nonzero with $F_{N}=1-T_{N}$. From our conductance value for the $v=2 \mathrm{QH}$ plateau, $T_{N} \sim 0.98$. The observed $F_{N} \sim 0.05$ is close to the theoretically expected Fano factor $\sim 0.02$. The slight deviation at smaller magnetic fields (1.4-1.8 T) could be due to a small bulk contribution at a large applied bias $(\sim 2 \mathrm{meV})$, which does not affect $F_{S}$ measured at a very small bias energy $(<0.2 \mathrm{meV})$. Further, we would like to note, in Ref. [22], we observed a significantly smaller enhancement of shot noise without a robust quantization of conductance in a bilayer graphene-niobium QH-SC system, thus not providing any insight into the AES dynamics.

Next, we discuss an effective model for AESs as well as microscopic NEGF calculations to understand the observation of quantized conductance with a large nonzero Fano factor in a graphene $\mathrm{QH}-\mathrm{SC}$ junction.

An effective model for AESs. An effective model of a QH-SC interface is schematically shown in Fig. 1(a) [21,39]. The zero-energy eigenstates of the AESs can be written in the electron-hole basis $\{|e\rangle,|h\rangle\}$ as $\left|\psi_{1}\right\rangle=\alpha|e\rangle+\beta|h\rangle$ and $\left|\psi_{2}\right\rangle=\beta^{*}|e\rangle-\alpha^{*}|h\rangle$ with wave vectors $k_{1}$ and $k_{2}$ along the edge and $|\alpha|^{2}+|\beta|^{2}=1$. By writing an incoming electronlike state as a linear combination of AES eigenstates, the Andreev reflection probability $\left(P_{\mathrm{AR}}\right)$ is obtained as $P_{\mathrm{AR}}=$ $4|\alpha|^{2}|\beta|^{2} \sin ^{2}(\phi / 2)$, where $\phi=\left(k_{1}-k_{2}\right) L$ is the phase difference acquired between the two AES eigenstates over the length $L$ (SM Sec. S1 [35]). When the AESs are neutral electron-hole hybrids, i.e., $|\alpha|^{2}=|\beta|^{2}=1 / 2, P_{\mathrm{AR}}(\phi)=$ $\sin ^{2}(\phi / 2)$. For $\phi=0,2 \pi$, no Cooper pair is transmitted due to the complete reflection of all the incident electrons. In the opposite limit, $\phi=\pi$, all the incident electrons transmit as a Cooper pair due to perfect Andreev reflection. For the $v=2$ filling factor, the conductance is zero in the former limit, and twice the normal state conductance, $4 e^{2} / h$, in the latter. In general, the junction conductance oscillates between these two limits if the phase difference is tuned either by carrier density or by a magnetic field. For a realistic junction, the presence of disorder and inelastic processes at the current carrying edge [40] will introduce dephasing and decoherence, making the phase differences random. For a complete phase averaging of the transmitted current with a uniform distribution of phase, the conductance of the QH-SC junction becomes exactly the same as that of a $\mathrm{QH}-\mathrm{NM}$ junction, i.e., $2 e^{2} / h$. Moreover, for a given phase difference $\phi$, the power spectral density of shot noise $\left(S_{I}\right)$ [27] can be written as $S_{I}(\phi)=2 e^{*} I_{t}\left[1-P_{\mathrm{AR}}(\phi)\right]=$ $8 e I \sin ^{2}(\phi / 2)\left[1-\sin ^{2}(\phi / 2)\right]$, with the Cooper pair charge $e^{*}=2 e$. For a complete phase averaging, $S_{I}=e I$. Thus, the effective model gives a Fano factor of half for a QH-SC 

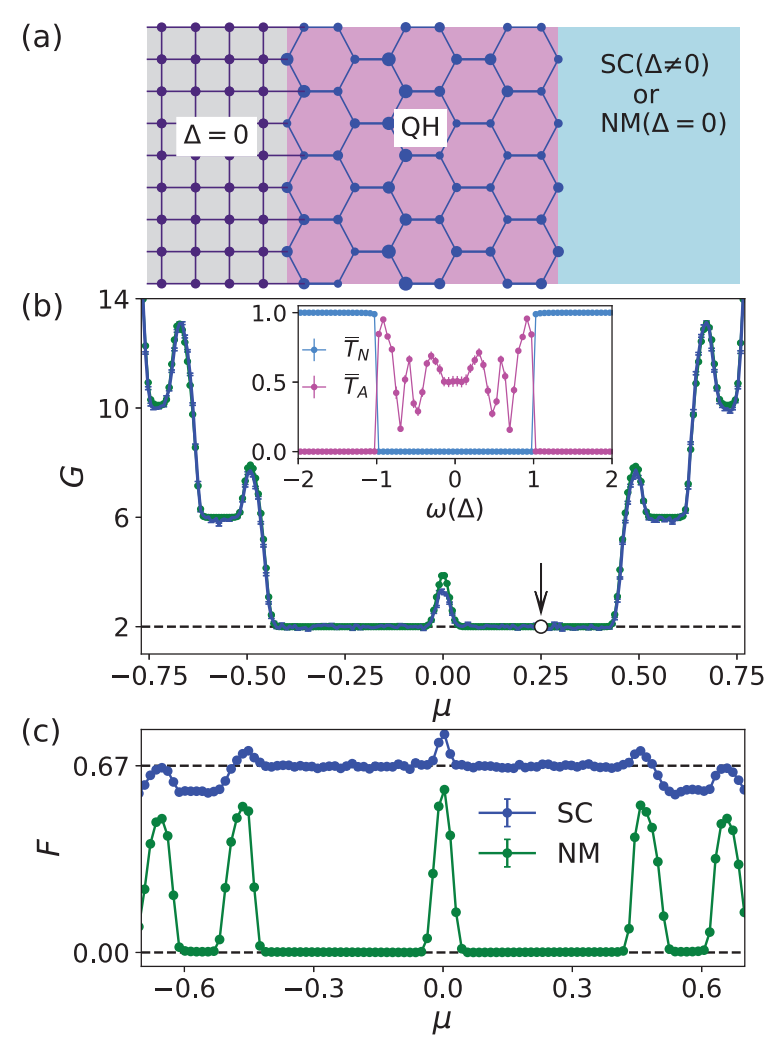

FIG. 4. (a) System for NEGF calculations. The graphene region has a nearest-neighbor hopping $t$ and magnetic field perpendicular to its plane, and the varying marker size of the lattice represents a particular realization of on-site disorder. (b) Conductance is plotted as a function of chemical potential for a disorder strength $W=0.5 t$, flux $\Phi=0.07$, and SC gap $\Delta=1 / 2750 t$. Conductance plateaus (blue) are realized by averaging over disorder and the plateaus appear at exactly the same conductance (green) expected for the QH-NM case. Inset: The normal and Andreev transmission coefficients for QH-SC are plotted as a function of excitation energy at the chemical potentials highlighted by the arrow. (c) Calculated Fano factor is plotted as a function of $\mu$ showing the 2/3 Fano factor at the $v=2$ plateau, which vanishes for the QH-NM case.

junction with perfectly quantized conductance plateaus, in agreement with our experimental observations.

NEGF calculations. For NEGF calculations, as in an earlier work [41], we consider an infinite strip of finite width having a hexagonal graphene lattice connected to two leads, one on either side [Fig. 4(a)]. Details of the model, parameters, and numerical calculations are given in SM Sec. S2 [35]. Within the NEGF formalism, the zero-temperature two-probe conductance (in units of $e^{2} / h$ ) in the linear response regime $(I=G V)$ for a small voltage bias $V$ is given by $G=2\left\langle\left[\operatorname{Tr}\left(T_{N}\right)+2 \operatorname{Tr}\left(T_{A}\right)\right]\right\rangle$, where $T_{N}$ and $T_{A}$ are normal and Adreev transmission matrices, respectively, for each spin species. The transmission matrices can be expressed in terms of the Green's function and self-energy of the leads (SM Sec. S2 [35]). Here, $\langle\cdots\rangle$ represents disorder averaging over many disorder realizations of $\left\{\epsilon_{i}\right\}$. We define the disorder averaged transmission matrices as $\bar{T}_{N(A)}=\left\langle\operatorname{Tr}\left(T_{N(A)}\right)\right\rangle$.

Figure 4(b) shows the disorder averaged conductance as a function of chemical potential $(\mu)$ with SC $(\Delta=1 / 2750 t)$ and normal $(\Delta=0)$ right leads. The conductance for both cases shows the same QH plateaus, as expected from the effective edge model. But, from the inset of Fig. 4(b), we can see that only Andreev transmission takes part in the transport, i.e., $\bar{T}_{N}=0$ and $\bar{T}_{A} \neq 0$, within the superconducting gap for the SC lead. Following Blanter et al. [27], the shot noise for a normal-superconductor junction is given by $S=$ $\left(16 e^{3} V / h\right) \operatorname{Tr}\left[T_{A}\left(1-T_{A}\right)\right]$, such that the Fano factor is $F=$ $2\left\langle\operatorname{Tr}\left[T_{A}\left(1-T_{A}\right)\right]\right\rangle /\left\langle\operatorname{Tr}\left[T_{A}\right]\right\rangle$. The numerically computed Fano factor for a graphene QH-SC junction is shown in Fig. 4(c) and compared with that of a QH-NM system. As is evident, the Fano factor for the conductance plateaus for QH-SC is nonzero, in contrast to the QH-NM system. In particular, $F=2 / 3$ for the $v=2$ plateau, unlike $F=1 / 2$ obtained from the edge model. This difference in values of $F$ from the NEGF calculations and the effective model can be traced back to the difference in the distribution $\operatorname{Tr} T_{A}$ over disorder realizations for the two models. As discussed in detail in SM Sec. S3 [35], in the case of NEGF calculations, we find that $\operatorname{Tr} T_{A}$ is uniformly distributed for $v=2$, thus producing $F=2 / 3$. On the contrary, in the effective model of AES in the preceding section, we assumed a uniform distribution of $\phi$ as opposed to the uniform distribution of $\sin ^{2}(\phi / 2) \propto T_{A}$. This difference between the NEGF calculation and effective model does not affect the conductance.

To conclude, our result of close to half a Fano factor along with quantized conductance for Andreev reflection dominated transport is an unambiguous signature of the existence of AESs at the QH-SC interface. The closer agreement of experimental results with the scenario of uniform phase averaging gives an indication of the crucial involvements of both static disorder and inelastic processes along the current-carrying edge even at very low temperatures [40]. Getting insights into such intriguing dynamical processes for AESs would be of great importance for the eventual realization of novel excitations, e.g., Majorana and parafermions, in various $\mathrm{QH}$ insulator-superconductor hybrid systems $[4,5,7,42,43]$.

\section{ACKNOWLEDGMENTS}

A.D. acknowledges support from the MHRD, Government of India under STARS research funding (STARS/APR2019/PS/156/FS), DST Nanomission (Grant No. DSTO2051), and also is thankful for support from Swarnajayanti Fellowship of the DST/SJF/PSA-03/2018-19.
[1] C. W. J. Beenakker, Rev. Mod. Phys. 87, 1037 (2015).

[2] W. Bishara and C. Nayak, Phys. Rev. Lett. 99, 066401 (2007).
[3] C. Nayak, S. H. Simon, A. Stern, M. Freedman, and S. Das Sarma, Rev. Mod. Phys. 80, 1083 (2008). 
[4] R. S. K. Mong, D. J. Clarke, J. Alicea, N. H. Lindner, P. Fendley, C. Nayak, Y. Oreg, A. Stern, E. Berg, K. Shtengel, and M. P. A. Fisher, Phys. Rev. X 4, 011036 (2014).

[5] X.-L. Qi, T. L. Hughes, and S.-C. Zhang, Phys. Rev. B 82, 184516 (2010).

[6] M. P. A. Fisher, Phys. Rev. B 49, 14550 (1994).

[7] P. San-Jose, J. L. Lado, R. Aguado, F. Guinea, and J. FernándezRossier, Phys. Rev. X 5, 041042 (2015).

[8] A. Rahmani and M. Franz, Rep. Prog. Phys. 82, 084501 (2019).

[9] J. Manousakis, C. Wille, A. Altland, R. Egger, K. Flensberg, and F. Hassler, Phys. Rev. Lett. 124, 096801 (2020).

[10] Y. Motome and J. Nasu, J. Phys. Soc. Jpn. 89, 012002 (2020).

[11] M. Kayyalha, D. Xiao, R. Zhang, J. Shin, J. Jiang, F. Wang, Y.-F. Zhao, R. Xiao, L. Zhang, K. M. Fijalkowski et al., Science 367, 64 (2020).

[12] H. Hoppe, U. Zülicke, and G. Schön, Phys. Rev. Lett. 84, 1804 (2000).

[13] M. Beconcini, M. Polini, and F. Taddei, Phys. Rev. B 97, 201403(R) (2018).

[14] P. Rickhaus, M. Weiss, L. Marot, and C. Schonenberger, Nano Lett. 12, 1942 (2012).

[15] M. B. Shalom, M. Zhu, V. Fal'ko, A. Mishchenko, A. Kretinin, K. Novoselov, C. Woods, K. Watanabe, T. Taniguchi, A. Geim et al., Nat. Phys. 12, 318 (2016).

[16] F. Amet, C. T. Ke, I. V. Borzenets, J. Wang, K. Watanabe, T. Taniguchi, R. S. Deacon, M. Yamamoto, Y. Bomze, S. Tarucha et al., Science 352, 966 (2016).

[17] G.-H. Park, M. Kim, K. Watanabe, T. Taniguchi, and H.-J. Lee, Sci. Rep. 7, 1 (2017).

[18] G.-H. Lee, K.-F. Huang, D. K. Efetov, D. S. Wei, S. Hart, T. Taniguchi, K. Watanabe, A. Yacoby, and P. Kim, Nat. Phys. 13, 693 (2017).

[19] M. R. Sahu, X. Liu, A. K. Paul, S. Das, P. Raychaudhuri, J. K. Jain, and A. Das, Phys. Rev. Lett. 121, 086809 (2018).

[20] A. Seredinski, A. W. Draelos, E. G. Arnault, M.-T. Wei, H. Li, T. Fleming, K. Watanabe, T. Taniguchi, F. Amet, and G. Finkelstein, Sci. Adv. 5, eaaw8693 (2019).

[21] L. Zhao, E. G. Arnault, A. Bondarev, A. Seredinski, T. F. Larson, A. W. Draelos, H. Li, K. Watanabe, T. Taniguchi, F. Amet et al., Nat. Phys. 16, 862 (2020).

[22] M. R. Sahu, A. K. Paul, A. Soori, K. Watanabe, T. Taniguchi, S. Mukerjee, and A. Das, Phys. Rev. B 100, 235414 (2019).

[23] G.-H. Lee and H.-J. Lee, Rep. Prog. Phys. 81, 056502 (2018).

[24] G. Finkelstein and F. Amet, Nat. Phys. 13, 625 (2017).

[25] T. Dvir, A. Zalic, E. H. Fyhn, M. Amundsen, T. Taniguchi, K. Watanabe, J. Linder, and H. Steinberg, Phys. Rev. B 103, 115401 (2021).

[26] Ö. Gül, Y. Ronen, S. Y. Lee, H. Shapourian, J. Zauberman, Y. H. Lee, K. Watanabe, T. Taniguchi, A. Vishwanath, A. Yacoby et al., arXiv:2009.07836.

[27] Y. M. Blanter and M. Büttiker, Phys. Rep. 336, 1 (2000).

[28] B.-R. Choi, A. E. Hansen, T. Kontos, C. Hoffmann, S. Oberholzer, W. Belzig, C. Schönenberger, T. Akazaki, and H. Takayanagi, Phys. Rev. B 72, 024501 (2005).

[29] A. B. Michelsen, T. L. Schmidt, and E. G. Idrisov, Phys. Rev. B 102, 125402 (2020).

[30] J. Tworzydło, B. Trauzettel, M. Titov, A. Rycerz, and C. W. J. Beenakker, Phys. Rev. Lett. 96, 246802 (2006).

[31] L. DiCarlo, J. R. Williams, Y. Zhang, D. T. McClure, and C. M. Marcus, Phys. Rev. Lett. 100, 156801 (2008).
[32] R. Danneau, F. Wu, M. F. Craciun, S. Russo, M. Y. Tomi, J. Salmilehto, A. F. Morpurgo, and P. J. Hakonen, Phys. Rev. Lett. 100, 196802 (2008).

[33] N. Kumada, F. Parmentier, H. Hibino, D. Glattli, and P. Roulleau, Nat. Commun. 6, 8068 (2015).

[34] S. Matsuo, S. Takeshita, T. Tanaka, S. Nakaharai, K. Tsukagoshi, T. Moriyama, T. Ono, and K. Kobayashi, Nat. Commun. 6, 8066 (2015).

[35] See Supplemental Material at http://link.aps.org/supplemental/ 10.1103/PhysRevB.104.L081404 for additional data on device fabrication and characterization, measurement techniques, and details of theoretical models used, which includes Refs. [4460].

[36] E. S. Tikhonov, D. V. Shovkun, M. Snelder, M. P. Stehno, Y. Huang, M. S. Golden, A. A. Golubov, A. Brinkman, and V. S. Khrapai, Phys. Rev. Lett. 117, 147001 (2016).

[37] J. D. Sau, R. M. Lutchyn, S. Tewari, and S. Das Sarma, Phys. Rev. B 82, 094522 (2010).

[38] A. K. Paul, M. R. Sahu, C. Kumar, K. Watanabe, T. Taniguchi, and A. Das, Commun. Phys. 3, 171 (2020).

[39] I. Khaymovich, N. Chtchelkatchev, I. Shereshevskii, and A. Mel'nikov, Europhys. Lett. 91, 17005 (2010).

[40] A. Marguerite, J. Birkbeck, A. Aharon-Steinberg, D. Halbertal, K. Bagani, I. Marcus, Y. Myasoedov, A. Geim, D. Perello, and E. Zeldov, Nature (London) 576, E6 (2019).

[41] Q.-f. Sun and X. Xie, J. Phys.: Condens. Matter 21, 344204 (2009).

[42] D. J. Clarke, J. Alicea, and K. Shtengel, Nat. Phys. 10, 877 (2014).

[43] J. Alicea and P. Fendley, Annu. Rev. Condens. Matter Phys. 7, 119 (2016).

[44] A. MacKinnon and B. Kramer, Z. Phys. B: Condens. Matter 53, 1 (1983).

[45] P. A. Lee and D. S. Fisher, Phys. Rev. Lett. 47, 882 (1981).

[46] J. A. Verges, Comput. Phys. Commun. 118, 71 (1999).

[47] X. Jehl, P. Payet-Burin, C. Baraduc, R. Calemczuk, and M. Sanquer, Phys. Rev. Lett. 83, 1660 (1999).

[48] K. Komatsu, C. Li, S. Autier-Laurent, H. Bouchiat, and S. Guéron, Phys. Rev. B 86, 115412 (2012).

[49] Y. Miyoshi, Y. Bugoslavsky, and L. F. Cohen, Phys. Rev. B 72, 012502 (2005).

[50] P. Zomer, M. Guimarães, J. Brant, N. Tombros, and B. Van Wees, Appl. Phys. Lett. 105, 013101 (2014).

[51] F. Pizzocchero, L. Gammelgaard, B. S. Jessen, J. M. Caridad, L. Wang, J. Hone, P. Bøggild, and T. J. Booth, Nat. Commun. 7, 11894 (2016).

[52] D. Purdie, N. Pugno, T. Taniguchi, K. Watanabe, A. Ferrari, and A. Lombardo, Nat. Commun. 9, 5387 (2018).

[53] Y. Ronen, Y. Cohen, J.-H. Kang, A. Haim, M.-T. Rieder, M. Heiblum, D. Mahalu, and H. Shtrikman, Proc. Natl. Acad. Sci. USA 113, 1743 (2016).

[54] S. K. Srivastav, M. R. Sahu, K. Watanabe, T. Taniguchi, S. Banerjee, and A. Das, Sci. Adv. 5, eaaw5798 (2019).

[55] H. B. Heersche, P. Jarillo-Herrero, J. B. Oostinga, L. M. Vandersypen, and A. F. Morpurgo, Nature (London) 446, 56 (2007).

[56] V. E. Calado, S. Goswami, G. Nanda, M. Diez, A. R. Akhmerov, K. Watanabe, T. Taniguchi, T. M. Klapwijk, and L. M. Vandersypen, Nat. Nanotechnol. 10, 761 (2015). 
[57] F. E. Schmidt, M. D. Jenkins, K. Watanabe, T. Taniguchi, and G. A. Steele, Nat. Commun. 9, 1 (2018).

[58] M. Zhu, M. B. Shalom, A. Mishchsenko, V. Falko, K. Novoselov, and A. Geim, Nanoscale 10, 3020 (2018).
[59] I. V. Borzenets, U. C. Coskun, H. T. Mebrahtu, Y. V. Bomze, A. I. Smirnov, and G. Finkelstein, Phys. Rev. Lett. 111, 027001 (2013).

[60] A. Rycerz, P. Recher, and M. Wimmer, Phys. Rev. B 80, 125417 (2009). 